Pacific Journal of Mathematics

ANALYTIC TWO-DIMENSIONAL SUBCENTER MANIFOLDS 


\title{
ANALYTIC TWO-DIMENSIONAL SUBCENTER MANIFOLDS FOR SYSTEMS WITH AN INTEGRAL
}

\author{
Al KeLley
}

For a real analytic system of ordinary differential equations with an integral $H$

$$
\begin{aligned}
H & =\frac{1}{2} \lambda\left(x^{2}+y^{2}\right)+F(z)+G(x, y, z) \\
\dot{x} & =\lambda y+X(x, y, z) \\
\dot{y} & =-\lambda x+Y(x, y, z) \\
\dot{z} & =B z+Z(x, y, z)
\end{aligned}
$$

where $x$ and $y$ are scalars; $z$ is an $m$-vector; $X, Y, Z$ are power series with no constant or linear terms; $B$ is a constant matrix with eigenvalues $\mu_{1}, \cdots, \mu_{m}$ and $i \lambda^{-1} \mu_{j} \neq$ integer $(j=$ $1, \cdots, m)$; the existence of a unique, local, real analytic, twodimensional, invariant subcenter manifold

$$
M^{\lambda}=\{(x, y, z)|| x|+| y \mid<\delta, z=w(x, y)\}
$$

is proved, where $w$ is a real analytic function with no constant or linear terms in its expansion about the origin. The manifold $M^{\lambda}$ is composed of a nested, one-parameter $(\rho \geqq 0)$ family of periodic orbits, and as $\rho \rightarrow 0$ the corresponding periodic orbit goes to the origin and its period goes to $2 \pi\left|\lambda^{-1}\right|$.

In this paper we extend the result of C. L. Siegel [7] to analytic systems of ordinary differential equations with an integral (see (1) below). C. L. Siegel proved Theorem 1 below with the additional restrictions that system (1) is Hamiltonian and that the matrix $B$ in (1) is a diagonal matrix. The removal of these two restrictions is a technical improvement; our emphasis is on the invariant manifold aspect of the theory (see Theorem 2 below). As far as proving the existence of a local, nested, one-parameter family of periodic orbits is concerned, the theorem proved by C. L. Siegel [7] is equivalent to a result of Liapounov [5] proved before the turn of the century. However, in addition C. L. Siegel proved that the invariant, twodimensional manifold comprising the nested, one-parameter family of periodic orbits is an analytic manifold, and that by means of an analytic change of variables, the differential equations on the invariant manifold have a very simple form. Using the proof method of Liapounov, one is able to conclude only that the invariant manifold of periodic orbits is continuous. (See A. Kelley [3] for a $C^{1}$ version of these ideas.) For real analytic systems with an integral we prove in $\S 4$ below that the invariant manifold is directly computable from the differential equations, and hence unique and real analytic. Since 
our manifold is imbedded in the center manifold (see A. Kelley [4]), we call it a subcenter manifold.

The convergence proof in Theorem 1 below is based on techniques developed in C. L. Siegel [7], J. Moser [6], and A. Kelley [1], [2].

2. Notation. Let $z=\left(z_{1}, \cdots, z_{p}\right)$ be a complex vector and let $q$ be a nonnegative integer. We define $\Gamma^{q}(z)$ to be the class of functions $\{F\}$ which satisfy (i), (ii), (iii) below.

(i) $F=F(z)$ is a complex scalar valued function, vector valued function, or matrix valued function (dim $F$ unspecified) defined for $z$ in some neighborhood of $z=0$.

(ii) $F$ has a convergent power series expansion in $z$ (components of $z$ ) about the point $z=0$; the coefficients in the expansion are either scalars, vectors, or matrices (as the case may be); there are no terms of degree less than $q$ in the expansion of $F$.

(iii) For some $K>0$ the components of $F$ are majorized by

$$
K\left(z_{1}+\cdots+z_{p}\right)^{q}\left[1-K\left(z_{1}+\cdots+z_{p}\right)\right]^{-1} .
$$

The constant $K$ depends on $F$.

Primes in this paper either designate majorants or designate variables and functions in a complex setting as contrasted to a real setting. Given $F(z) \in \Gamma^{q}(z)$ there exists $F^{\prime}(z)$ such that

$$
F(z) \ll F^{\prime}(z)
$$

where the symbol $\ll$ means $F$ and $F^{\prime}$ belong to the same $\Gamma$ class, $\operatorname{dim} F=\operatorname{dim} F^{\prime}$, and $F$ is majorized componentwise by $F^{\prime}$ in the sense of Cauchy.

The norm $|\cdot|$ is the euclidean norm on vectors and the operator norm on matrices. If $F=F(z)$ is a vector valued function of the vector $z$, then $F_{z}$ represents the usual Jacobian matrix of partial derivatives.

If $F \in \Gamma^{0}(x, y)$ where $x$ and $y$ are scalar variables, then $[F]_{\alpha, \beta}$ represents the coefficient of the $x^{a} y^{\beta}$ term in the expansion of $F$.

Let $F \in \Gamma^{0}(x, y)$ be a vector valued, real analytic function of the scalar variables $x$ and $y$, and let

$$
F(x, y)=\sum_{\alpha, \beta=0}^{\infty} F_{\alpha, \beta} x^{a} y^{\beta} .
$$

In $\S 4$ below we will use the notation

$$
F_{n}=[F]_{n}=\left(F_{n, 0}, F_{n-1,1}, \cdots, F_{0, n}\right) .
$$

Thus $F_{n}$ is a vector in $R^{(n+1) \operatorname{dim} F}$.

Throughout this paper $R=R(p, q)$ is used to designate an 
anylytic function, although occassionally (as above) the notation $z \in R^{n}$ is used to indicate that $z$ is an $n$-vector. The distinction will always be clear from the context.

3. Analytic changes of variables. Consider the complex analytic system of ordinary differential equations with an integral $H$

$$
\begin{aligned}
H(x, y, z) & =i \lambda x y+F(z)+G(x, y, z) \\
\dot{x} & =i \lambda x+X(x, y, z) \\
\dot{y} & =-i \lambda y+Y(x, y, z) \\
\dot{z} & =B z+Z(x, y, z)
\end{aligned}
$$

where $x$ and $y$ are complex variables; $z$ is a complex $m$-vector variable; $\lambda$ is a nonzero complex number; (in the most interesting case $\lambda$ is real, see (19) below for the real counterpart to system (1)); $B$ is a constant matrix in Jordan canonical form; $B=B^{0}+B^{1}$,

$$
\begin{aligned}
& B^{0}=\operatorname{diag}\left(\mu_{1}, \cdots, \mu_{m}\right) \\
& B^{1}=\operatorname{subdiag}\left(\delta_{1}, \cdots, \delta_{m-1}\right)
\end{aligned}
$$

with $\delta_{j}=0$ or $\delta, \delta>0$ sufficiently small (to be specified below); $X, Y, Z \in \Gamma^{2}(x, y, z) ; \operatorname{dim} X=\operatorname{dim} x$, etc.; $F$ is a quadratic form in the components of $z$ ( $F \equiv 0$ is allowed); $G \in \Gamma^{3}(x, y, z)$ (which implies that $\left.H \in \Gamma^{2}(x, y, z)\right) ; H$ is an integral for system (1).

THEOREm 1. If $i \lambda^{-1} \mu_{j} \neq$ integer $(j=1, \cdots, m)$, then for system (1) there exists a change of variables (not unique)

$$
\begin{aligned}
& x=p+P(p, q) \\
& y=q+Q(p, q) \\
& z=r+R(p, q)
\end{aligned}
$$

such that

$$
\begin{aligned}
\dot{p} & =i \lambda(1+a(p q)) p+\widetilde{P}(p, q, r) \\
\dot{q} & =-i \lambda(1+a(p q)) q+\widetilde{Q}(p, q, r) \\
\dot{r} & =B r+\widetilde{R}(p, q, r) \\
\widetilde{P}, \widetilde{Q}, \widetilde{R} & \equiv 0 \text { when } r=0,
\end{aligned}
$$

where $a=a(p q)$ is a function of the product $p q ; a(\omega) \in \Gamma^{1}(\omega)$ (which implies $\left.a(p q) \in \Gamma^{2}(p, q)\right) ; P, Q, R \in \Gamma^{2}(p, q) ; \widetilde{P}, \widetilde{Q}, \widetilde{R} \in \Gamma^{2}(p, q, r)$.

Proof. First we construct a formal change of variables (2) such that 


$$
\begin{aligned}
\dot{p} & =i \lambda(1+a(p q))+\widetilde{P}(p, q, r) \\
\dot{q} & =-i \lambda(1+\widetilde{a}(p q))+\widetilde{Q}(p, q, r) \\
\dot{r} & =B r+\widetilde{R}(p, q, r) \\
\widetilde{P}, \widetilde{Q}, \widetilde{R} & \equiv 0 \text { when } r=0,
\end{aligned}
$$

where $a$ and $\tilde{a}$ are formal power series in the product $p q$ with no constant terms. Then we will use the fact that $H$ is an integral for system (1) to prove that $\widetilde{a}=a$ as formal power series. Finally, convergence will be proved using the Cauchy method of majorants.

If such a change of variables (2) exists, then from (1), (2), (4) we obtain

$$
\begin{aligned}
& \dot{P}=-i \lambda a p+i \lambda P+X(p+P, q+Q, r+R)-\widetilde{P}(p, q, r) \\
& \dot{Q}=i \lambda \widetilde{a} q-i \lambda Q+Y(p+P, q+Q, r+R)-\widetilde{Q}(p, q, r) \\
& \dot{R}=B R+Z(p+P, q+Q, r+R)-\widetilde{R}(p, q, r)
\end{aligned}
$$

where $P=P(p, q)$, etc. Taking the derivative on the left side of (5) implicity and using the condition $\widetilde{P}, \widetilde{Q}, \widetilde{R} \equiv 0$ when $r=0$, we obtain

$$
\begin{aligned}
& p P_{p}-q P_{q}-P \\
= & -a p-a p P_{p}+\tilde{a} q P_{q}-i \lambda^{-1} X(p+P, q+Q, R) \\
& p Q_{p}-q Q_{q}+Q \\
(6) \quad= & \tilde{a} q-a p Q_{p}+\tilde{a} q Q_{q}-i \lambda^{-1} Y(p+P, q+Q, R) \\
& p \frac{\partial}{\partial p} R_{j}-q \frac{\partial}{\partial q} R_{j}+i \lambda^{-1} \mu_{j} R_{j} \\
= & -i \lambda^{-1} \delta_{j-1} R_{j-1}-a p \frac{\partial}{\partial p} R_{j}+\widetilde{a} q \frac{\partial}{\partial q} R_{j}-i \lambda^{-1} Z_{j}(p+P, q+Q, R) \\
& \quad(j=1, \cdots, m)
\end{aligned}
$$

where $\delta_{0}=0$. From (6) the coefficients of $P, Q, R, a, \widetilde{a}$ can be computed recursively. Let

$$
P(p, q)=\sum_{\alpha+\beta \geqq 2} P_{\alpha, \beta} p^{a} q^{\beta}
$$

etc. From (6) we have

$$
\begin{aligned}
& \sum(\alpha-\beta-1) P_{\alpha, \beta} p^{a} q^{\beta}=-\sum a_{\beta, \beta} p^{\beta+1} q^{\beta}+\cdots \\
& \sum(\alpha-\beta+1) Q_{\alpha, \beta} p^{a} q^{\beta}=\sum \widetilde{a}_{\alpha, \alpha} p^{a} q^{a+1}+\cdots \\
& \sum\left(\alpha-\beta+i \lambda^{-1} \mu_{j}\right) R_{j, \alpha, \beta}=\cdots \quad(j=1, \cdots, m) .
\end{aligned}
$$

If we equate corresponding coefficients on the left and right side of (6) (equivalently (7)), we obtain 


$$
\begin{aligned}
& (\alpha-\beta-1) P_{\alpha, \beta} \\
= & {\left[-a p-a p P_{p}+\tilde{a} q P_{q}-i \lambda^{-1} X(p+P, q+Q, R)\right]_{\alpha, \beta} } \\
& (\alpha-\beta+1) Q_{\alpha, \beta} \\
= & {\left[\widetilde{a} q-a p Q_{p}+\tilde{a} q Q_{q}-i \lambda^{-1} Y(p+P, q+Q, R)\right]_{\alpha, \beta} } \\
& \left(\alpha-\beta+i \lambda^{-1} \mu_{j}\right) R_{j, \alpha, \beta} \\
= & {\left[-i \lambda^{-1} \delta_{j-1} R_{j-1}-\alpha p \frac{\partial}{\partial p} R_{j}+\tilde{a} q \frac{\partial}{\partial q} R_{j}-i \lambda^{-1} Z_{j}(p+P, q+Q, R)\right]_{\alpha, \beta} }
\end{aligned}
$$

Clearly, the expressions on the right side of (8) involve only the coefficients of $P, Q, R$ of order less than $\alpha+\beta$ and the coefficient of $R_{1}, \cdots, R_{j-1}$ of order less than or equal to $\alpha+\beta$. When $\alpha=\beta+1$, then $a_{\beta, \beta}$ must be chosen so that the right side of the first equation in (8) vanishes. Similarly when $\beta=\alpha+1$, then $\widetilde{a}_{\alpha, \alpha}$ must be chosen so that the right side of the second equation in (8) vanishes. By hypothesis $i \lambda^{-1} \mu_{j} \neq$ integer $(j=1, \cdots, m)$. Therefore

$$
\alpha-\beta+i \lambda^{-1} \mu_{j} \neq 0 \quad(j=1, \cdots, m)
$$

and all the coefficients of $R$ can be computed recursively from (8). All the coefficients of $P$ and $Q$ can be computed recursively, except $P_{\beta+1, \beta}$ and $Q_{\alpha, \alpha+1}(\alpha, \beta=1,2, \cdots)$ which can be chosen arbitrarily. Let

$$
\begin{aligned}
& \hat{P}(p, q)=\sum_{\beta=1}^{\infty} \hat{P}_{\beta+1, \beta} p^{\beta+1} q^{\beta} \\
& \hat{Q}(p, q)=\sum_{\alpha=1}^{\infty} \hat{Q}_{\alpha, \alpha+1} p^{a} q^{\alpha+1}
\end{aligned}
$$

by any given convergent power series (for example $\hat{P}, \hat{Q} \equiv 0$ ), and define

$$
\begin{aligned}
& P_{\beta+1, \beta}=\hat{P}_{\beta+1, \beta} \\
& Q_{\alpha, \alpha+1}=\hat{Q}_{\alpha, \alpha+1}
\end{aligned} \quad(\alpha, \beta=1,2, \cdots) .
$$

Now, from (8) and (9) the coefficients of $P, Q, R, a, \tilde{a}$ can be computed recursively.

Next we want to show that $a$ and $\tilde{a}$ are equal as formal power series. Since $H$ is an integral for (1),

$$
\widetilde{H}(p, q)=H(p+P, q+Q, R)
$$

is a (formal) integral for system (3) restricted to the manifold given by $r=0$; namely for the system

$$
\begin{aligned}
& \dot{p}=i \lambda(1+a) p \\
& \dot{q}=-i \lambda(1+\widetilde{a}) q .
\end{aligned}
$$


Therefore, operating only in the sense of formal power series, we obtain

$$
\frac{d}{d t} \widetilde{H}=\widetilde{H}_{p} i \lambda(1+a) p-\widetilde{H}_{q} i \lambda(1+\widetilde{a}) q=0,
$$

or equivalently

$$
p \widetilde{H}_{p}-q \widetilde{H}_{q}=-a p \widetilde{H}_{p}+\widetilde{a} q \widetilde{H}_{q} .
$$

Let

$$
\widetilde{H}(p, q)=\sum_{\alpha+\beta \geqq 2} \widetilde{H}_{\alpha, \beta} p^{a} q^{\beta} .
$$

Equating coefficients in (10), we have

$$
(\alpha-\beta) \widetilde{H}_{\alpha, \beta}=\left[-a p \widetilde{H}_{p}+\widetilde{a} q \breve{H}_{q}\right]_{\alpha, \beta} .
$$

Since $a=a(p q)$ and $\widetilde{a}=\widetilde{a}(p q)$ are power series in the product $p q$, it follows from (11) by induction that $\widetilde{H}_{\alpha, \beta}=0$ for all $\alpha \neq \beta$. Therefore $\widetilde{H}$ is a (formal) power series in the product $p q$, and this implies

$$
p \widetilde{H}_{p}=q \widetilde{H}_{q} \text {. }
$$

Hence from (10)

$$
(a-\widetilde{a}) p \widetilde{H}_{p}=0
$$

Since

$$
p \widetilde{H}_{p}=i \lambda p q+\cdots
$$

is a nontrivial power series, we conclude that as formal power series

$$
a(p q)=\widetilde{a}(p q) \text {. }
$$

Equation (6) can now be written

$$
\begin{aligned}
& p P_{p}-q P_{q}-P=-a p-a\left\{p P_{p}-q P_{q}\right\}-i \lambda^{-1} X(p+P, q+Q, R) \\
& p Q p-q Q_{q}+Q=a q-a\left\{p Q_{p}-q Q_{q}\right\}-i \lambda^{-1} Y(p+P, q+Q, R) \\
& p R_{p}-q R_{q}+i \lambda^{-1} B^{0} R \\
& =-i \lambda^{-1} B^{1} R-a\left\{p R_{p}-q R_{q}\right\}-i \lambda^{-1} Z(p+P, q+Q, R) .
\end{aligned}
$$

Let $X^{\prime}, Y^{\prime}, Z^{\prime}, \hat{P}^{\prime}, \hat{Q}^{\prime}, a^{\prime}$ be the smallest majorants of

$$
i \lambda^{-1} X, i \lambda^{-1} Y, i \lambda^{-1} Z, \hat{P}, \hat{Q}, a
$$

respectively. Thus

$$
X^{\prime}, Y^{\prime}, Z^{\prime} \in \Gamma^{2}(x, y, z) ; \hat{P}^{\prime}, \hat{Q}^{\prime} \in \Gamma^{2}(p, q) .
$$

The power series $a^{\prime}$ must still be shown to be convergent. Define 


$$
\begin{aligned}
& P^{\prime}(p, q)=\sum\left(|\alpha-\beta|+\delta_{\alpha \beta}\right)\left|P_{\alpha, \beta}\right| p^{a} q^{\beta} \\
& Q^{\prime}(p, q)=\sum\left(|\alpha-\beta|+\delta_{\alpha \beta}\right)\left|Q_{\alpha, \beta}\right| p^{a} q^{\beta} \\
& R_{j}^{\prime}(p, q)=\sum\left(|\alpha-\beta|+\delta_{\alpha \beta}\right)\left|R_{j, \alpha, \beta}\right| p^{a} q^{\beta} \quad(j=1, \cdots, m) \\
& R^{\prime}=\left(R_{1}^{\prime}, \cdots, R_{m}^{\prime}\right)
\end{aligned}
$$

where $\delta_{\alpha \beta}$ is the Kronecker delta. Clearly

$$
P \ll P^{\prime} ; \quad Q \ll Q^{\prime} ; \quad R \ll R^{\prime} .
$$

Choose $K>0$ such that for all nonnegative integers $\alpha$ and $\beta$ (except as noted)

$$
\begin{array}{lll}
|\alpha-\beta-1|^{-1}\left(|\alpha-\beta|+\delta_{\alpha \beta}\right) \leqq K & (\alpha-\beta-1 \neq 0) \\
|\alpha-\beta+1|^{-1}\left(|\alpha-\beta|+\delta_{\alpha \beta}\right) \leqq K & (\alpha-\beta+1 \neq 0) \\
\left|\alpha-\beta+i \lambda^{-1} \mu_{j}\right|^{-1}\left(|\alpha-\beta|+\delta_{\alpha \beta}\right) \leqq K & (j=1, \cdots, m) .
\end{array}
$$

From (12), (13), (14) it follows that

$$
\begin{aligned}
P^{\prime} & \ll K\left[a^{\prime} P^{\prime}+X^{\prime}\left(p+P^{\prime}, q+Q^{\prime}, R^{\prime}\right)\right]+\hat{P}^{\prime} \\
Q^{\prime} & \ll K\left[a^{\prime} Q^{\prime}+Y^{\prime}\left(p+P^{\prime}, q+Q^{\prime}, R^{\prime}\right)\right]+\hat{Q}^{\prime} \\
R^{\prime} & \ll K\left[\left|\lambda^{-1}\right| B^{1} R^{\prime}+a^{\prime} R^{\prime}+Z^{\prime}\left(p+P^{\prime}, q+Q^{\prime}, R^{\prime}\right)\right] \\
a^{\prime} p & \ll a^{\prime} P^{\prime}+X^{\prime}\left(p+P^{\prime}, q+Q^{\prime}, R^{\prime}\right) .
\end{aligned}
$$

Since $P^{\prime}, Q^{\prime}, R^{\prime}, a^{\prime}$ all have real, nonnegative coefficients, it is sufficient to prove convergence when $p=q=\zeta$. Let

$$
\begin{aligned}
& P^{\prime}(\zeta, \zeta)=\zeta P^{\prime \prime}(\zeta) ; Q^{\prime}(\zeta, \zeta)=\zeta Q^{\prime \prime}(\zeta) ; R^{\prime}(\zeta, \zeta)=\zeta R^{\prime \prime}(\zeta) ; \\
& \hat{P}^{\prime}(\zeta, \zeta)=\zeta \hat{P}^{\prime \prime}(\zeta) ; \hat{Q}^{\prime}(\zeta, \zeta)=\zeta \hat{Q}^{\prime \prime}(\zeta) ; a^{\prime}\left(\zeta^{2}\right)=a^{\prime \prime}(\zeta) .
\end{aligned}
$$

From (15) we have

$$
\begin{aligned}
& P^{\prime \prime} \ll K\left[a^{\prime \prime} P^{\prime \prime}+\zeta^{-1} X^{\prime}\left(\zeta+\zeta P^{\prime \prime}, \zeta+\zeta Q^{\prime \prime}, \zeta R^{\prime \prime}\right)\right]+\hat{P}^{\prime \prime} \\
& Q^{\prime \prime} \ll K\left[a^{\prime \prime} Q^{\prime \prime}+\zeta^{-1} Y^{\prime}\left(\zeta+\zeta P^{\prime \prime}, \zeta+\zeta Q^{\prime \prime}, \zeta R^{\prime \prime}\right)\right]+\hat{Q}^{\prime \prime} \\
& R^{\prime \prime} \ll K\left[\left|\lambda^{-1}\right| B^{1} R^{\prime \prime}+a^{\prime \prime} R^{\prime \prime}+\zeta^{-1} Z^{\prime}\left(\zeta+\zeta P^{\prime \prime}, \zeta+\zeta Q^{\prime \prime}, \zeta R^{\prime \prime}\right)\right] \\
& a^{\prime \prime} \ll a^{\prime \prime} P^{\prime \prime}+\zeta^{-1} X^{\prime}\left(\zeta+\zeta P^{\prime \prime}, \zeta+\zeta Q^{\prime \prime}, \zeta R^{\prime \prime}\right) .
\end{aligned}
$$

Define formal power series $P^{*}=P^{*}(\zeta), P^{*}(0)=0$, etc., by the functional equations

$$
\begin{aligned}
& P^{*}=K\left[a^{*} P^{*}+\zeta^{-1} X^{\prime}\left(\zeta+\zeta P^{*}, \zeta+\zeta Q^{*}, \zeta R^{*}\right)\right]+\hat{P}^{\prime \prime} \\
& Q^{*}=K\left[a^{*} Q^{*}+\zeta^{-1} Y^{\prime}\left(\zeta+\zeta P^{*}, \zeta+\zeta Q^{*}, \zeta R^{*}\right)\right]+\hat{Q}^{\prime \prime} \\
& R^{*}=K\left[\left|\lambda^{-1}\right| B^{1} R^{*}+a^{*} R^{*}+\zeta^{-1} Z^{\prime}\left(\zeta+\zeta P^{*}, \zeta+\zeta Q^{*}, \zeta R^{*}\right)\right] \\
& a^{*}=a^{*} P^{*}+\zeta^{-1} X^{\prime}\left(\zeta+\zeta P^{*}, \zeta+\zeta Q^{*}, \zeta R^{*}\right) .
\end{aligned}
$$

Comparing (17) with (16) it follows that 


$$
P^{\prime \prime} \ll P^{*} ; Q^{\prime \prime} \ll Q^{*} ; R^{\prime \prime} \ll R^{*} ; a^{\prime \prime} \ll a^{*} .
$$

But the convergence of $P^{*}, Q^{*}, R^{*}, a^{*}$ in (17) follows from the implicit function theorem provided $\delta$ in $B^{1}$ is chosen sufficiently small to insure that

$$
K\left|\lambda^{-1} B^{1}\right|<1
$$

Thus from

$$
P^{*}, Q^{*}, R^{*}, a^{*} \in \Gamma^{1}(\zeta)
$$

we conclude that

$$
P, Q, R, a \in \Gamma^{2}(p, q) \text {. }
$$

To show that

$$
\widetilde{P}, \widetilde{Q}, \widetilde{R} \in \Gamma^{2}(p, q, r)
$$

write equation (5) as

$$
\begin{aligned}
\widetilde{P}= & -i \lambda a p+i \lambda P+X(p+P, q+Q, r+R) \\
& -P_{p}\{i \lambda(1+a) p+\widetilde{P}\}-P_{q}\{-i \lambda(1+a) q+\widetilde{Q}\} \\
\widetilde{Q}= & i \lambda a q-i \lambda Q+Y(p+P, q+Q, r+R) \\
& -Q_{p}\{i \lambda(1+a) p+\widetilde{P}\}-Q_{q}\{-i \lambda(1+a) q+\widetilde{Q}\} \\
\widetilde{R}= & B R+Z(p+P, q+Q, r+R) \\
& -R_{p}\{i \lambda(1+a) p+\widetilde{P}\}-R_{q}\{-i \lambda(1+a) q+\widetilde{Q}\} .
\end{aligned}
$$

Since $P, Q, R, a$ are known analytic functions of $(p, q)$, we can regard (18) as an equation in the variables $p, q, r, \widetilde{P}, \widetilde{Q}, \widetilde{R}$ and use the implicit function Theorem to solve for $\widetilde{P}=\widetilde{P}(p, q, r)$, etc. Clearly

$$
\widetilde{P}, \widetilde{Q}, \widetilde{R} \equiv 0 \text { when } r=0 \text {. }
$$

This completes the proof of Theorem 1.

4. Real analytic invariant manifolds. For real analytic systems of equations with an integral, we want to show the existence of real analytic invariant manifolds. Theorem 1 will be the prime tool for this program.

Consider the real analytic system of ordinary differential equations with an integral $H$

$$
\begin{aligned}
& H(x, y, z)=\frac{1}{2} \lambda\left(x^{2}+y^{2}\right)+F(z)+G(x, y, z) \\
& \dot{x}=\lambda y+X(x, y, z) \\
& \dot{y}=-\lambda x+Y(x, y, z) \\
& \dot{z}=B z+Z(x, y, z)
\end{aligned}
$$


where $x$ and $y$ are real scalar variables; $z$ is a real $m$-vector variable; $\lambda$ is a nonzero real number; $B$ is a real, constant matrix with eigenvalues $\mu_{1}, \cdots, \mu_{m} ; X, Y, Z \in \Gamma^{2}(x, y, z)$ are real analytic; $\operatorname{dim} X=$ $\operatorname{dim} x$, etc.; $F$ is a real quadratic form in the components of $z$; $G \in \Gamma^{3}(x, y, z)$ is real analytic; $H$ is a real analytic integral for (1).

THEOREM 2. If $i \lambda^{-1} \mu_{j} \neq$ integer $(j=1, \cdots, m)$, then for system (19) there exists a unique, local, real analytic, two-dimensional, invariant manifold

$$
M^{\lambda}=\{(x, y, z)|| x|+| y \mid<\delta, z=w(x, y)\}
$$

where $w \in \Gamma^{2}(x, y)$ is real analytic and is directly computable (thus it is not necessary to reduce the differential equations to a normal form such as (38) below in order to compute $\left.M^{\lambda}\right)$; moreover, the manifold $M^{\lambda}$ is composed of a nested, one-parameter $(\rho \geqq 0)$ family of periodic orbits, and as $\rho \rightarrow 0$ the corresponding periodic orbit goes to the origin and its period goes to $2 \pi\left|\lambda^{-1}\right|$.

Proof. The second part of this theorem is relatively easy to prove. Suppose the real analytic function $w$ which defines $M^{\lambda}$ is known. Then

$$
\widetilde{H}(x, y)=\frac{1}{2} \lambda\left(x^{2}+y^{2}\right)+F(w(x, y))+G(x, y, w(x, y))
$$

is a real analytic integral for (1) restricted to $M^{\lambda}$. Introduce

$$
\begin{aligned}
& x=\rho(1+b) \cos \theta \\
& y=\rho(1+b) \sin \theta
\end{aligned}
$$

into the equation

$$
\widetilde{H}(x, y)=\frac{1}{2} \lambda \rho^{2}
$$

to obtain

$$
b+\frac{1}{2} b^{2}+\rho^{-2} \lambda^{-1}(\widetilde{F}+\widetilde{G})=0
$$

where

$$
\begin{aligned}
\widetilde{F} & =\widetilde{F}(\theta, \rho, b)=F(w(\rho(1+b) \cos \theta, \rho(1+b) \sin \theta)) \\
\widetilde{G} & =\widetilde{G}(\theta, \rho, b)=G(\rho(1+b) \cos \theta, \cdots) .
\end{aligned}
$$

We observe that $\rho^{-2} \lambda^{-1}(\widetilde{F}+\widetilde{G})$ is well defined for $\rho=0$. From (20) by means of the implicit function theorem we can solve for $b=b(\theta, \rho)$ with $b$ having period $2 \pi$ in $\theta$ and $b(\theta, 0) \equiv 0$, so that 


$$
\begin{aligned}
& x=\rho(1+b(\theta, \rho)) \cos \theta \\
& y=\rho(1+b(\theta, \rho)) \sin \theta \\
& z=w(\rho(1+b(\theta, \rho)) \cos \theta, \rho(1+b(\theta, \rho)) \sin \theta)
\end{aligned}
$$

is a representation of the one-parameter $(\rho \geqq 0)$ family of nested periodic orbits which comprise $M^{\lambda}$.

Continuing in this manner, it is not difficult to prove the assertion concerning the periods of these periodic orbits. See [3] for the details.

For a more complete analysis of the differential equations (19) restricted to $M^{\lambda}$, one can show the existence of a real analytic change of variables

$$
\begin{aligned}
& x=p+P(p, q) \\
& y=q+Q(p, q) \\
& z=r+R(p, q)
\end{aligned}
$$

such that on $M^{2}$ (given by $z=R(p, q)$ )

$$
\begin{aligned}
& \dot{p}=\lambda\left(1+a\left(p^{2}+q^{2}\right)\right) q \\
& \dot{q}=-\lambda\left(1+a\left(p^{2}+q^{2}\right)\right) p
\end{aligned}
$$

where $P, Q, R, a \in \Gamma^{2}(p, q)$ are real analytic; $a$ is a real power series in the quadratic form $p^{2}+q^{2}$. In this formulation the periodicity of the solutions of (21) is obvious (the circles $p^{2}+q^{2}=\rho^{2}$ in the $(p, q)$-plane are orbits) and the assertion concerning the periods of these solutions is immediate. The details of this analysis are given in $\S 5$ below.

We now prove the first part of Theorem 2. Primes for the rest of this section will denote variables and functions in a complex setting as opposed to unprimed variables and functions in a real setting. Introduce the change of variables

$$
\begin{aligned}
& x=\frac{1}{\sqrt{2}}\left(i x^{\prime}+y^{\prime}\right) \\
& y=\frac{1}{\sqrt{2}}\left(-x^{\prime}-i y^{\prime}\right) \\
& z=\widetilde{B} z^{\prime}
\end{aligned}
$$

so that system (19) transforms to

$$
\begin{aligned}
& H^{\prime}\left(x^{\prime}, y^{\prime}, z^{\prime}\right)=i \lambda x^{\prime} y^{\prime}+F^{\prime}\left(z^{\prime}\right)+G\left(x^{\prime}, y^{\prime}, z^{\prime}\right) \\
& \dot{x}^{\prime}=i \lambda x^{\prime}+X^{\prime}\left(x^{\prime}, y^{\prime}, z^{\prime}\right) \\
& \dot{y}^{\prime}=-i \lambda y^{\prime}+Y^{\prime}\left(x^{\prime}, y^{\prime}, z^{\prime}\right) \\
& \dot{z}^{\prime}=B^{\prime} z^{\prime}+Z^{\prime}\left(x^{\prime}, y^{\prime}, z^{\prime}\right)
\end{aligned}
$$


where

$$
H^{\prime}\left(x^{\prime}, y^{\prime}, z^{\prime}\right)=H\left(\frac{1}{\sqrt{2}}\left(i x^{\prime}+y^{\prime}\right), \frac{1}{\sqrt{2}}\left(-x^{\prime}-i y^{\prime}\right), \widetilde{B} z^{\prime}\right) ;
$$

$B^{\prime}=\widetilde{B}^{-1} B \widetilde{B}$ is in Jordan canonical form; $X^{\prime}, Y^{\prime}, Z^{\prime}$ are related to $X, Y, Z$ in the usual way. Theorem 1 asserts the existence of an invariant manifold

$$
\begin{aligned}
M^{\prime \lambda}=\left\{\left(x^{\prime}, y^{\prime}, z^{\prime}\right) \mid x^{\prime}\right. & =p^{\prime}+P^{\prime}\left(p^{\prime}, q^{\prime}\right), \\
y^{\prime} & \left.=q^{\prime}+Q^{\prime}\left(p^{\prime}, q^{\prime}\right), z^{\prime}=R^{\prime}\left(p^{\prime}, q^{\prime}\right)\right\}
\end{aligned}
$$

where $P^{\prime}, Q^{\prime}, R^{\prime} \in \Gamma^{2}\left(p^{\prime}, q^{\prime}\right)$. But the change of variables

$$
\begin{aligned}
& x^{\prime}=p^{\prime}+P^{\prime}\left(p^{\prime}, q^{\prime}\right) \\
& y^{\prime}=q^{\prime}+Q^{\prime}\left(p^{\prime}, q^{\prime}\right)
\end{aligned}
$$

can be inverted to give

$$
\begin{aligned}
& p^{\prime}=p^{\prime}\left(x^{\prime}, y^{\prime}\right)=x^{\prime}+\cdots \\
& q^{\prime}=q^{\prime}\left(x^{\prime}, y^{\prime}\right)=y^{\prime}+\cdots
\end{aligned}
$$

so that

$$
M^{\prime \lambda}=\left\{\left(x^{\prime}, y^{\prime}, z^{\prime}\right)|| x^{\prime}|+| y^{\prime} \mid<\delta, z^{\prime}=w^{\prime}\left(x^{\prime}, y^{\prime}\right)\right\}
$$

where

$$
w^{\prime}\left(x^{\prime}, y^{\prime}\right)=R^{\prime}\left(p^{\prime}\left(x^{\prime}, y^{\prime}\right), q^{\prime}\left(x^{\prime}, y^{\prime}\right)\right),
$$

which implies $w^{\prime} \in \Gamma^{2}\left(x^{\prime}, y^{\prime}\right)$. With the representation given by (24) it is not clear that $M^{\prime 2}$ is unique, but with the representation (25) we can easily show uniqueness for $M^{\prime \prime}$. Since the manifold is invariant,

$$
\dot{w}^{\prime}=B^{\prime} w^{\prime}+Z^{\prime}\left(x^{\prime}, y^{\prime}, w^{\prime}\right)
$$

equivalently

$$
\begin{aligned}
& i \lambda x^{\prime} w_{x^{\prime}}^{\prime}-i \lambda y^{\prime} w_{y^{\prime}}^{\prime}-B^{\prime} w^{\prime} \\
= & Z^{\prime}\left(x^{\prime}, y^{\prime}, w^{\prime}\right)-w_{x^{\prime}}^{\prime} X^{\prime}\left(x^{\prime}, y^{\prime}, w^{\prime}\right)-w_{y^{\prime}}^{\prime} Y^{\prime}\left(x^{\prime}, y^{\prime}, w^{\prime}\right) .
\end{aligned}
$$

Expanding $w^{\prime}$ as a power series and equating coefficients in (26), we have

$$
\begin{aligned}
& \left(i \lambda(\alpha-\beta) I-B^{\prime}\right) w_{\alpha, \beta}^{\prime} \\
= & {\left[Z^{\prime}\left(x^{\prime}, y^{\prime}, w^{\prime}\right)-w_{x^{\prime}}^{\prime} X^{\prime}\left(x^{\prime}, y^{\prime}, w^{\prime}\right)-w_{y^{\prime}}^{\prime} Y^{\prime}\left(x^{\prime}, y^{\prime}, w^{\prime}\right)\right]_{\alpha, \beta} }
\end{aligned}
$$

where $I$ is the identity matrix. Since by hypothesis $i \lambda^{-1} \mu_{j} \neq$ integer $(j=1, \cdots, m)$ where $\mu_{1}, \cdots, \mu_{m}$ are the eigenvalues of $B^{\prime}$, it follows that 


$$
\operatorname{det}\left(i \lambda(\alpha-\beta) I-B^{\prime}\right) \neq 0 \text {. }
$$

Since the right side of (27) depends only on the coefficients of $w^{\prime}$ having order less than $\alpha+\beta$, we conclude that $w^{\prime}$ is uniquely determined by (27) and that the manifold $M^{\prime \prime}$ is unique. Define

$$
w(x, y)=\widetilde{B} w^{\prime}\left(\frac{1}{\sqrt{2}}(-i x-y), \frac{1}{\sqrt{2}}(x+i y)\right) .
$$

Then

$$
\dot{w}=B w+Z(x, y, w)
$$

equivalently

$$
\begin{aligned}
& \lambda y w_{x}-\lambda x w_{y}-B w \\
= & Z(x, y, w)-w_{x} X(x, y, w)-w_{y} Y(x, y, w) .
\end{aligned}
$$

The function $w$ given by (28) satisfies (30). Suppose $w^{*} \in \Gamma^{2}(x, y)$ is another solution to (30). Then $w^{*}$ determines a function $w^{* \prime} \in \Gamma^{2}\left(x^{\prime}, y^{\prime}\right)$ given by

$$
w^{* \prime}\left(x^{\prime}, y^{\prime}\right)=\widetilde{B}^{-1} w^{*}\left(\frac{1}{\sqrt{2}}\left(i x^{\prime}+y^{\prime}\right), \frac{1}{\sqrt{2}}\left(-x^{\prime}-i y^{\prime}\right)\right) .
$$

But since $w^{*}$ satisfies (30), it also satisfies (29). Then from (31) we conclude that $w^{* \prime}$ satisfies (26), hence $w^{* \prime} \equiv w^{\prime}$ and because (28) and (31) are inverse relations to each other, $w^{*} \equiv w$. Therefore we conclude that (30) has a unique solution belonging to the class $\Gamma^{2}(x, y)$.

It remains to be shown that $w$ is a real analytic function. If we expand the left side of (30) in a power series, we obtain

$$
\begin{aligned}
& \lambda y w_{x}-\lambda x w_{y}-B w \\
= & \sum \lambda \alpha w_{\alpha, \beta} x^{a-1} y^{\beta+1}-\sum \lambda \beta w_{\alpha, \beta} x^{a+1} y^{\beta-1}-\sum B w_{\alpha, \beta} x^{a} y^{\beta} \\
= & \sum\left\{\lambda(\alpha+1) w_{\alpha+1, \beta-1}-\lambda(\beta+1) w_{\alpha-1, \beta+1}-B w_{\alpha, \beta}\right\} x^{a} y^{\beta} .
\end{aligned}
$$

For any power series

$$
f=\sum f_{\alpha, \beta} x^{a} y^{\beta}
$$

define

$$
f_{n}=[f]_{n}=\left[\sum f_{\alpha, \beta} x^{a} y^{\beta}\right]_{n}=\left(f_{n, 0}, f_{n-1,1}, \cdots, f_{0, n}\right)
$$

for all nonnegative integers $n$. Thus if $f$ is a vector, then $\operatorname{dim} f_{n}=$ $(n+1) \operatorname{dim} f$. If $f$ and $f^{\prime}$ are two power series related by

$$
\begin{aligned}
& f^{\prime}\left(x^{\prime}, y^{\prime}\right)=f(x, y)=f\left(\frac{1}{\sqrt{2}}\left(i x^{\prime}+y^{\prime}\right), \frac{1}{\sqrt{2}}\left(-x^{\prime}-i y^{\prime}\right)\right) \\
& f(x, y)=f^{\prime}\left(x^{\prime}, y^{\prime}\right)=f^{\prime}\left(\frac{1}{\sqrt{2}}(-i x-y), \frac{1}{\sqrt{2}}(x+i y)\right),
\end{aligned}
$$


then for each nonnegative integer $n$ there exists a matrix $L_{n}$, $\operatorname{det} L_{n} \neq 0$, such that

$$
f_{n}^{\prime}=L_{n} f_{n} \text {. }
$$

Clearly the matrix $L_{n}$ depends only on $\operatorname{dim} f$; other than this $L_{n}$ is independent of $f$.

Now, from (32) we obtain

$$
\left[\lambda y w_{x}-\lambda x w_{y}-B w\right]_{n}=B_{n} w_{n} \quad(n=2,3, \cdots)
$$

where

$$
\begin{aligned}
B_{n}= & \text { super-diag }(-\lambda I,-2 \lambda I, \cdots,-n \lambda I) \\
& +\operatorname{diag}(-B,-B, \cdots,-B) \\
& +\operatorname{sub-diag}(n \lambda I,(n-1) \lambda I, \cdots, \lambda I) .
\end{aligned}
$$

with $I=$ identity matrix, $\operatorname{dim} I=\operatorname{dim} B$. In a similar fashion we obtain

$$
\left[i \lambda x^{\prime} w_{x^{\prime}}^{\prime}-i \lambda y^{\prime} w_{y^{\prime}}^{\prime}-B^{\prime} w^{\prime}\right]_{n}=B_{n}^{\prime} w_{n}^{\prime} \quad(n=2,3, \cdots)
$$

where

$$
B_{n}^{\prime}=\operatorname{diag}\left(i n \lambda I-B^{\prime}, i(n-2) \lambda I-B^{\prime}, i(n-4) \lambda I-B^{\prime}, \cdots,-i n \lambda I-B^{\prime}\right) \text {. }
$$

Since $\operatorname{det}\left(i k \lambda I-B^{\prime}\right) \neq 0$ for any integer $k$, it follows that

$$
\operatorname{det} B_{n}^{\prime} \neq 0
$$$$
(n=2,3, \cdots) \text {. }
$$

A straight forward calculation shows that

$$
\lambda y \frac{\partial}{\partial x}-\lambda x \frac{\partial}{\partial y}=i \lambda x^{\prime} \frac{\partial}{\partial x^{\prime}}-i \lambda y^{\prime} \frac{\partial}{\partial y^{\prime}},
$$

and from this fact along with (28) we conclude

$$
\begin{aligned}
& \lambda y w_{x}-\lambda x w_{y}-B w=\widetilde{B}\left\{i \lambda x^{\prime} w_{x^{\prime}}^{\prime}-i \lambda y^{\prime} w_{y^{\prime}}^{\prime}-B^{\prime} w\right\} \\
& {\left[\lambda y w_{x}-\lambda x w_{y}-B w\right]_{n}=L_{n}^{-1}\left[\widetilde{B}\left\{i \lambda x^{\prime} w_{x^{\prime}}^{\prime}-i \lambda y^{\prime} w_{y^{\prime}}^{\prime}-B^{\prime} w^{\prime}\right\}\right]_{n}} \\
& B_{n} w_{n}=L_{n}^{-1} \widetilde{B}_{n} B_{n}^{\prime} w_{n}^{\prime}=L_{n}^{-1} \widetilde{B}_{n} B_{n}^{\prime} L_{n} w_{n},
\end{aligned}
$$

where

$$
\widetilde{B}_{n}=\operatorname{diag}(\widetilde{B}, \widetilde{B}, \cdots, \widetilde{B}) .
$$

Since (34) can be shown valid for all $w_{n} \in R^{(n+1) \text { dim } w}$, it follows that

$$
\begin{aligned}
& B_{n}=L_{n}^{-1} \widetilde{B}_{n} B_{n}^{\prime} L_{n} \\
& \operatorname{det} B_{n}=\operatorname{det} \widetilde{B}_{n} \cdot \operatorname{det} B_{n}^{\prime} \neq 0 .
\end{aligned}
$$


From (30) and (33) we obtain

$$
\begin{array}{r}
B_{n} w_{n}=\left[Z(x, y, w)-w_{x} X(x, y, w)-w_{y} Y(x, y, w)\right]_{n} \\
(n=2,3, \cdots) .
\end{array}
$$

Finally, from (35) and (36) we conclude the coefficients of $w$ are determined recursively from (30), or equivalently (36), and since $B_{n}(n=2,3, \cdots)$ is a real matrix, $B_{n}^{-1}$ is also real and $w \in \Gamma^{2}(x, y)$ is real analytic. Thus we have shown that the function $w$ which defines the manifold

$$
M^{\lambda}=\{(x, y, z)|| x|+| y \mid<\delta, z=w(x, y)\}
$$

is uniquely and recursively determined from (30), (35), (36) as a real power series. The fact that $w \in \Gamma^{2}(x, y)$ is guaranteed by the existence of the real analytic integral $H$. This completes the proof of Theorem 2.

5. Real analytic changes of variables. In this section for a real analytic system of equations we construct a real analytic change of variables.

Theorem 3. If $i \lambda^{-1} \mu_{j} \neq$ integer $(j=1, \cdots, m)$ then for system (19) there exists a real analytic change of variables (not unique)

$$
\begin{aligned}
& x=p+P(p, q) \\
& y=q+Q(p, q) \\
& z=r+R(p, q)
\end{aligned}
$$

such that

$$
\begin{aligned}
& \dot{p}=\lambda\left(1+a\left(p^{2}+q^{2}\right)\right) q+\widetilde{P}(p, q, r) \\
& \dot{q}=-\lambda\left(1+a\left(p^{2}+q^{2}\right)\right) p+\widetilde{Q}(p, q, r) \\
& \dot{r}=B r+\widetilde{R}(p, q, r) \\
& \widetilde{P}, \widetilde{Q}, \widetilde{R} \equiv 0 \text { when } r=0,
\end{aligned}
$$

where $a=a\left(p^{2}+q^{2}\right)$ is a real analytic function of the quadratic form $p^{2}+q^{2}$ with no constant term in its expansion about the origin; $P, Q, R \in \Gamma^{2}(p, q)$ are real analytic; $\widetilde{P}, \widetilde{Q}, \widetilde{R} \in \Gamma^{2}(p, q, r)$ are real analytic.

Proof. By means of the change of variables (22) we obtain system (23). From Theorem 1 we can find a change of variables 


$$
\begin{aligned}
& x^{\prime}=p^{\prime}+P^{\prime}\left(p^{\prime}, q^{\prime}\right) \\
& y^{\prime}=q^{\prime}+Q^{\prime}\left(p^{\prime}, q^{\prime}\right) \\
& z^{\prime}=r^{\prime}+R^{\prime}\left(p^{\prime}, q^{\prime}\right)
\end{aligned}
$$

such that

$$
\begin{aligned}
& \dot{p}^{\prime}=i \lambda\left(1+a^{\prime}\left(p^{\prime} q^{\prime}\right)\right) p^{\prime}+\widetilde{P}^{\prime}\left(p^{\prime}, q^{\prime}, r^{\prime}\right) \\
& \dot{q}^{\prime}=-i \lambda\left(1+a^{\prime}\left(p^{\prime} q^{\prime}\right)\right) q^{\prime}+\widetilde{Q}^{\prime}\left(p^{\prime}, q^{\prime}, r^{\prime}\right) \\
& \dot{r}^{\prime}=B^{\prime} r^{\prime}+\widetilde{R}^{\prime}\left(p^{\prime}, q^{\prime}, r^{\prime}\right) \\
& \widetilde{P}^{\prime}, \widetilde{Q}^{\prime}, \widetilde{R}^{\prime} \equiv 0 \text { when } r^{\prime}=0
\end{aligned}
$$

where $a^{\prime}=a^{\prime}\left(p^{\prime} q^{\prime}\right)$ is a convergent power series in the product $p^{\prime} q^{\prime}$ with no constant term; $P^{\prime}, Q^{\prime}, R^{\prime} \in \Gamma^{2}\left(p^{\prime}, q^{\prime}\right) ; \widetilde{P}, \widetilde{Q}, \widetilde{R} \in \Gamma^{2}\left(p^{\prime}, q^{\prime}, r^{\prime}\right)$. In the construction of the change of variables (39) one must restrict the choice of power series $\hat{P}^{\prime}, \hat{Q}^{\prime}$ (see (8) and (9)) to be transformations via (22) of real power series. We will make this condition explicit in (43) below. Define

$$
\begin{aligned}
& p=\frac{1}{\sqrt{2}}\left(i p^{\prime}+q^{\prime}\right) \\
& q=\frac{1}{\sqrt{2}}\left(-p^{\prime}-i q^{\prime}\right) \\
& r=\widetilde{B} r^{\prime} .
\end{aligned}
$$

Then from (22), (39), (41) we obtain the change of variables (37) where

$$
\begin{aligned}
a\left(p^{2}+q^{2}\right)= & a^{\prime}\left(-\frac{i}{2}\left(p^{2}+q^{2}\right)\right) \\
P(p, q)= & \frac{1}{\sqrt{2}} i P^{\prime}\left(\frac{1}{\sqrt{2}}(-i p-q), \frac{1}{\sqrt{2}}(p+i q)\right) \\
& +\frac{1}{\sqrt{2}} Q^{\prime}\left(\frac{1}{\sqrt{2}}(-i p-q), \frac{1}{\sqrt{2}}(p+i q)\right) \\
Q(p, q)= & -\frac{1}{\sqrt{2}} P^{\prime}\left(\frac{1}{\sqrt{2}}(-i p-q), \frac{1}{\sqrt{2}}(p+i q)\right) \\
& -\frac{1}{\sqrt{2}} i Q^{\prime}\left(\frac{1}{\sqrt{2}}(-i p-q), \frac{1}{\sqrt{2}}(p+i q)\right) \\
R(p, q)= & \widetilde{B} R^{\prime}\left(\frac{1}{\sqrt{2}}(-i p-q), \frac{1}{\sqrt{2}}(p+i q)\right) .
\end{aligned}
$$

In particular we require $\hat{P}, \hat{Q}$ given by 


$$
\begin{aligned}
\hat{P}(p, q)= & \frac{1}{\sqrt{2}} i \hat{P}^{\prime}\left(\frac{1}{\sqrt{2}}(-i p-q), \frac{1}{\sqrt{2}}(p+i q)\right) \\
& +\frac{1}{\sqrt{2}} \hat{Q}^{\prime}\left(\frac{1}{\sqrt{2}}(-i p-q), \frac{1}{\sqrt{2}}(p+i q)\right) \\
\hat{Q}(p, q)= & -\frac{1}{\sqrt{2}} \hat{P}^{\prime}\left(\frac{1}{\sqrt{2}}(-i p-q), \frac{1}{\sqrt{2}}(p+i q)\right) \\
& -\frac{1}{\sqrt{2}} i \hat{Q}^{\prime}\left(\frac{1}{\sqrt{2}}(-i p-q), \frac{1}{\sqrt{2}}(p+i q)\right)
\end{aligned}
$$

to be real analytic functions of $(p, q)$. The functions $\widetilde{P}, \widetilde{Q}, \widetilde{R}$ are given by formulas similar to those for $P, Q, R$ in (42).

It remains to show that $a, P, Q, R, \widetilde{P}, \widetilde{Q}, \widetilde{R}$ are real analytic functions. From (19), (37), (38) we obtain

$$
\begin{aligned}
& q P_{p}-p P_{q}-Q=-a q-a\left\{q P_{p}-p P_{q}\right\}+\lambda^{-1} X(p+P, q+Q, R) \\
& q Q_{p}-p Q_{q}+P=a p-a\left\{q Q_{p}-p Q_{q}\right\}+\lambda^{-1} Y(p+P, q+Q, R) \\
& q R_{p}-p R_{q}-\lambda^{-1} B R=-a\left\{q R_{p}-p R_{q}\right\}+\lambda^{-1} Z(p+P, q+Q, R) .
\end{aligned}
$$

Following a procedure analogous to the computation of $w$ in (30) we can compute the coefficients of $a, P, Q, R$ recursively from (44). Since $X, Y, Z$ are real analytic and $\lambda$ is a real, nonzero scalar, it follows that $a, P, Q, R$ are real analytic. This completes the proof of Theorem 3.

\section{BIBLIOGRAPHY}

1. A. Kelley, Changes of variables near a periodic orbit, Trans. Amer. Math. Soc. 126 (1967), 316-334.

2. - Changes of variables near a periodic surface or invariant manifold, Trans. Amer. Math. Soc. 131 (1968), 356-364.

3. - On the Liapounov subcenter manifold, J. Math. Anal. Appl. 18 (1967), $472-478$.

4. - The stable, center-stable, center, center-unstable, unstable manifolds, J. Differential Equations 3 (1967), 546-570.

5. A. Liapounov, Probleme General de la Stabilite du Mouvement, Ann. Math. Studies No. 17, Princeton Univ. Press, Princeton, 1949, 375-392.

6. J. Moser, On the generalization of a theorem of A. Liapounoff, Comm. Pure Appl. Math. 11 (1958), 257-271.

7. C. L. Siegel, Vorlesungen uber Himmelsmechanik, Springer-Verlag, Berlin, (1956), 82-92.

Received May 10, 1968. Research partly sponsored by the National Academy of Sciences and by the Air Force Office of Scientific Research, Office of Aerospace Research, United States Air Force, under AFOSR Grant No. 1317-67.

University of California, Santa Cruz 


\section{PACIFIC JOURNAL OF MATHEMATICS}

\section{EDITORS}

H. ROYDEN
Stanford University
Stanford, California

\section{R. R. PHELPS}

University of Washington

Seattle, Washington 98105
J. DUGUNDJI

Department of Mathematics

University of Southern California

Los Angeles, California 90007

\section{RICHARD ARENS}

University of California

Los Angeles, California 90024

\section{ASSOCIATE EDITORS}

E. F. BeCKenbaCh
B. H. NEUMANN

F. WOLF
K. YoshidA

\section{SUPPORTING INSTITUTIONS}

\author{
UNIVERSITY OF BRITISH COLUMBIA \\ CALIFORNIA INSTITUTE OF TECHNOLOGY \\ UNIVERSITY OF CALIFORNIA \\ MONTANA STATE UNIVERSITY \\ UNIVERSITY OF NEVADA \\ NEW MEXICO STATE UNIVERSITY \\ OREGON STATE UNIVERSITY \\ UNIVERSITY OF OREGON \\ OSAKA UNIVERSITY \\ UNIVERSITY OF SOUTHERN CALIFORNIA
}

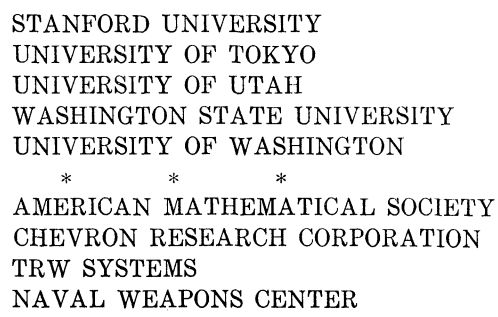

The Supporting Institutions listed above contribute to the cost of publication of this Journal, but they are not owners or publishers and have no responsibility for its content or policies.

Mathematical papers intended for publication in the Pacific Journal of Mathematics should be in typed form or offset-reproduced, double spaced with large margins. Underline Greek letters in red, German in green, and script in blue. The first paragraph or two must be capable of being used separately as a synopsis of the entire paper. It should not contain references to the bibliography. Manuscripts, in duplicate if possible, may be sent to any one of the four editors. Please classify according to the scheme of Math. Rev. 36, 1539-1546. All other communications to the editors should be addressed to the managing editor, Richard Arens, University of California, Los Angeles, California, 90024.

50 reprints are provided free for each article; additional copies may be obtained at cost in multiples of 50 .

The Pacific Journal of Mathematics is published monthly. Effective with Volume 16 the price per volume (3 numbers) is $\$ 8.00$; single issues, $\$ 3.00$. Special price for current issues to individual faculty members of supporting institutions and to individual members of the American Mathematical Society: $\$ 4.00$ per volume; single issues $\$ 1.50$. Back numbers are available.

Subscriptions, orders for back numbers, and changes of address should be sent to Pacific Journal of Mathematics, 103 Highland Boulevard, Berkeley, California, 94708.

PUBLISHED BY PACIFIC JOURNAL OF MATHEMATICS, A NON-PROFIT CORPORATION

Printed at Kokusai Bunken Insatsusha (International Academic Printing Co., Ltd.), 7-17, Fujimi 2-chome, Chiyoda-ku, Tokyo, Japan. 


\section{Pacific Journal of Mathematics \\ Vol. 29, No. $2 \quad$ June, 1969}

Bruce Langworthy Chalmers, On boundary behavior of the Bergman kernel function and related domain functionals ................... 243

William Eugene Coppage, Peirce decomposition in simple Lie-admissible power-associative rings .............................. 251

Edwin Duda, Compactness of mappings...................... 259

Earl F. Ecklund Jr., On prime divisors of the binomial coefficient......... 267

Don E. Edmondson, A modular topological lattice ............... 271

Phillip Alan Griffith, A note on a theorem of Hill ................... 279

Marcel Herzog, On finite groups with independent cyclic Sylow

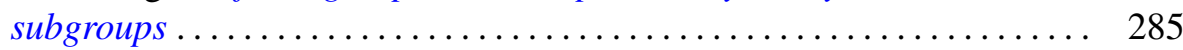

James A. Huckaba, Extensions of pseudo-valuations................. 295

S. A. Huq, Semivarieties and subfunctors of the identity functor ........ 303

I. Martin (Irving) Isaacs and Donald Steven Passman, Finite groups with small character degrees and large prime divisors. II ............ 311

Carl Kallina, A Green's function approach to perturbations of periodic

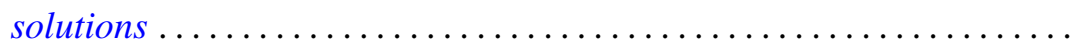

$\mathrm{Al}$ (Allen Frederick) Kelley, Jr., Analytic two-dimensional subcenter manifolds for systems with an integral ....................

Alistair H. Lachlan, Initial segments of one-one degrees ............ 351

Marion-Josephine Lim, Rank k Grassmann products ............. 367

Raymond J. McGivney and William Henry Ruckle, Multiplier algebras of

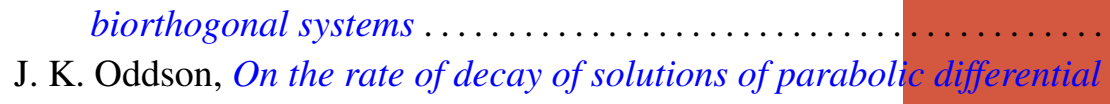

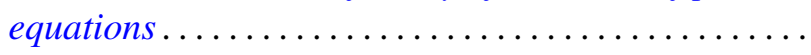

Helmut R. Salzmann, Geometries on surfaces ........... .

Annemarie Schlette, Artinian, almost abelian groups and their groups of automorphisms ............................

Edgar Lee Stout, Additional results on modules over polydisc algebras ...

Lajos Tamássy, A characteristic property of the sphere . .

Mark Lawrence Teply, Some aspects of Goldie's torsion theory. ...

Freddie Eugene Tidmore, Extremal structure of star-shaped sets ...

461

Leon Jarome Weill, Unconditional and shrinking bases in locally convex spaces... 